\title{
Asymptotic methods for delay equations
}

\author{
A.C. FOWLER \\ Mathematical Institute, Oxford University, 24-29 St Giles', Oxford OX1 3LB, England(fowler@maths.ox.ac.uk)
}

Received 11 November 2004; accepted in revised form 31 August 2005

\begin{abstract}
Asymptotic methods for singularly perturbed delay differential equations are in many ways more challenging to implement than for ordinary differential equations. In this paper, four examples of delayed systems which occur in practical models are considered: the delayed recruitment equation, relaxation oscillations in stem cell control, the delayed logistic equation, and density wave oscillations in boilers, the last of these being a problem of concern in engineering two-phase flows. The ways in which asymptotic methods can be used vary from the straightforward to the perverse, and illustrate the general technical difficulties that delay equations provide for the central technique of the applied mathematician.
\end{abstract}

Key words: delayed logistic equation, delayed recruitment equation, density-wave oscillation, SIR model, stem cell dynamics

\section{Introduction}

Delay equations, also known as differential-delay equations (DDE) or functional differential equations (FDE), are differential equations in which the dependent variable appears with one or more retarded arguments. The type example (with a single delay), is the first-order DDE

$$
\dot{x}=f\left(x, x_{\tau}\right),
$$

where $\dot{x}=\mathrm{d} x / \mathrm{d} t, x \in \mathbf{R}^{n}$, and we use the notation $x_{\tau}$ to indicate the delayed argument:

$$
x_{\tau} \equiv x(t-\tau)
$$

Such equations arise in practice where there is a transport delay between different compartments, or where there is a gestation period involved in the system. Many of these kinds of system are biological or physiological in origin.

Delay equations are notoriously difficult to analyse. This largely stems from the fact that the presence of the delay makes even a single scalar differential delay equation an infinitedimensional system, which can, for example, be thought of as an evolution equation for a function in a suitable Banach space. A simple way to see this is to consider the initial-value problem for (1.1). It is fairly evident that initial data is required on an interval:

$$
x=\phi(s) \quad \text { for } s \in[-\tau, 0] .
$$

It is also fairly obvious that armed with continuous initial data, Picard's theorem and a Lipschitz continuous function $f$, we can integrate forward an interval $\tau$ at a time to obtain a unique solution. This is called the method of steps (e.g., [1, p. 226]), and shows that the DDE (1.1) is equivalent to a discrete map on the infinite-dimensional Banach space $B=C[-\tau, 0]$ of 
continuous functions on the interval $[-\tau, 0]$. This is the Poincare map for the equation, and it is infinite-dimensional.

A more abstract setting is to view (1.1) directly as an ordinary differential equation on $B$. We consider $X_{t}(s) \in B$ to be a continuous function with $s \in[-\tau, 0]$, which depends on $t$. Specifically, we take $X_{t}(s) \equiv x(t+s)$, and define the operator $N$ on $B$ as

$$
N[\phi(s)] \equiv f[\phi(0), \phi(-\tau)] \quad \text { for } \phi \in B ;
$$

then the DDE (1.1) can be written as the infinite-dimensional ordinary differential equation on $B$ :

$$
\dot{X}_{t}=N\left[X_{t}\right] .
$$

The use of the subscript notation in (1.5) is distinct from that in (1.1), which is unfortunate, but both are commonly used in their respective contexts. In the remainder of this paper, we use the subscript notation as in (1.1).

The complexity of delay differential equations means that there are very few hard results. The literature on the subject thus tends to focus on functional analytic methods (e.g., [2, 3]). Often the delay is a time delay, and the DDE represents the evolution of a dynamical system. Linear stability results are then of interest, and can be obtained by solving algebraic equations of the form $g(\sigma)=0$, where $\sigma$ is the growth rate. Whereas $g$ is a polynomial for an ordinary differential equation, it is transcendental for DDE, involving exponential terms which arise from the delays. This makes solution for $\sigma$ more awkward, but still (at least for simple systems) feasible, as we show below.

The present paper concerns the practical application of asymptotic methods to delay equations. Whereas for ordinary differential equations, there are numerous expository books available (e.g., $[4,5])$, this is not true for delay differential equations, essentially because the delay appears to upset the methodological application of perturbation methods.

Broadly speaking, perturbation methods come in two varieties: multiple-scales methods, often for initial-value problems having oscillatory solutions, and boundary-layer methods, often for boundary-value problems. The application of multiple-scales methods to DDE is fairly straightforward (e.g., [6, 7]), and we will not dwell on these.

However, the application of boundary-layer-type methods causes severe difficulties, and it is these difficulties which we aim to highlight. Singular perturbations arise when the delay is large, and in such situations, asymptotic methods can appear intractable despite the applied mathematician's philosophy that where there is an $\varepsilon$, there is yet hope. In this paper, we survey particular problems in which a delay arises in a realistic model, and we discuss the ways in which asymptotic methods can (or cannot) work.

\section{Chaos in the delayed recruitment equation}

We begin our survey with one of the hardest problems I know, which illustrates some of the typical behaviour of delay equations, and also (graphically) the basic difficulties that asymptotic methods for delay differential equations face.

The delayed recruitment equation

$$
\varepsilon \dot{x}=-x+\mu f\left(x_{1}\right),
$$

where $x_{1}=x(t-1)$, is a relatively simple model which can be used to model the evolution of a resource-limited population with a significant gestational delay in reproduction [8]. In 
this equation, as applied to populations, $-x$ is the natural mortality rate, and $\mu f\left(x_{1}\right)$ is the resource-dependent reproduction rate, supposed dependent on the population at an earlier time because of gestation period. Similar models arise in models of physiological control, notably in respiration and in the control of blood-cell production [9].

In (2.1), $\varepsilon$ represents the ratio of the mortality time scale to the delay, and $\mu$ represents the strength of the regeneration term. In models of physiological control, $f$ is usually a positive monotonically decreasing function. In population dynamics, it would be a positive concave unimodal (i.e., with one maximum) function. In both cases, there will be a unique positive steady state $x^{*}$ where $x^{*}=\mu f\left(x^{*}\right)$ (if $f$ is unimodal, this is true if we suppose $f^{\prime}(0)>1$ ).

At this steady state, let us suppose that $f^{\prime}\left(x^{*}\right)<0$ (this is the usual case). If the delay were zero, then the steady state would be asymptotically stable, but the delay allows the possibility of instability. Specifically, if we linearise about $x^{*}$, the resulting linear perturbation equation has solutions $\propto \exp (\sigma t)$, where $\sigma$ is determined by the transcendental equation

$$
\sigma=-\alpha-\Gamma \mathrm{e}^{-\sigma},
$$

where

$$
\alpha=\frac{1}{\varepsilon}, \quad \Gamma=\frac{\mu\left|f^{\prime}\left(x^{*}\right)\right|}{\varepsilon} .
$$

Instability occurs as a Hopf bifurcation when $\Gamma>\Gamma_{1}(\alpha)$, where $\Gamma_{1}(\alpha)$ is a positive monotone increasing function of $\alpha$, with $\Gamma_{1}(0)=\frac{1}{2} \pi, \Gamma_{1} \sim \alpha$ as $\alpha \rightarrow \infty$ (e.g., [3, p. 305]).

Thus, for large negative slope of $f$, periodic solutions occur. Usually these bifurcate stably, and near criticality, their form can be computed via multiple-scales methods in the usual way (because the centre manifold is of finite dimension two).

The question of interest in the present context is what happens dynamically when $\varepsilon$ is small, and the oscillations become strongly nonlinear. 'Classical' theory for ordinary differential equations would suggest that the oscillations become relaxational in this limit, as happens for the Van der Pol oscillator [5, Section 2.6], and indeed this is suggested by the form of (2.1), which appears to be of singular-perturbation form when $\varepsilon \ll 1$.

Proceeding naïvely, we obtain an outer solution to (2.1) (away from rapid transition regions) by putting $\varepsilon=0$, thus

$$
x \approx \mu f\left(x_{1}\right) .
$$

This is simply a difference equation. For an arbitrary continuous initial function $x=x_{0}(s), s \in$ $[-1,0],(2.4)$ defines a piecewise smooth function $x(t)$ for $t>0$, with discontinuities at $t=0, t=1$, etc. We would thus expect or hope that the solutions of the DDE would consist of solutions of the functional map (2.4) joined by regions in which $x$ changes rapidly. As we shall see, sometimes this is the case, and sometimes not.

To investigate this further, let us consider two particular forms for $f$, the logistic function

$$
f(x)=x(1-x),
$$

and the Hill function (e.g., [10, p. 13])

$$
f(x)=h(x)=\frac{1}{1+x^{n}} .
$$

The exponent $n$ is greater than one, and for illustration we will take $n=3$.

Both forms of $f$ give a unique positive steady state (for the logistic function this is if $\mu>1$ ). As $\mu$ increases, the fixed point of the map (2.4) becomes unstable, and a period two cycle occurs. 
For the logistic map (but not the Hill function), further increase of $\mu$ leads to further perioddoubling, and eventually chaos. An obvious conjecture is that, if the underlying map (2.4) has a stable period two orbit $\left(x_{1}, x_{2}, x_{1}, \ldots\right)$, for example, then the differential delay equation (2.1) has a (stable) relaxational periodic orbit which jumps rapidly (on a time scale of $O(\varepsilon)$ ) between the two values $x_{1}$ and $x_{2}$.

Figure 1 shows a numerical solution of (2.1) using the Hill-function recruitment function (2.6). This shows the typical relaxational form of the oscillator. So-called slowly varying oscillations of this type have been studied by Mallet-Paret and his co-workers (see, for example, [11], and more recently [12]). This solution confirms that stable periodic solutions of period near two do exist, and a formalism to describe them is given below.

More generally, we might suppose, or hope, that if the underlying map (2.4) has a solution $\left(x_{1}, x_{2}, x_{3}, \ldots\right)$, then a slowly varying solution of the differential delay equation might exist consisting of the same sequence of plateaux, connected by $\varepsilon$-thick boundary layers. Doubt of this is raised by the fact that, if the map is chaotic, then any such sequence is unstable.

In fact, the behaviour of the differential delay equation is quite different in this case. Figure 2 shows the solution of (2.1) when the recruitment function is taken to be the logistic function (2.5), at a parameter value where the underlying map is chaotic. Clearly the solution of the differential delay equation is also, but the solution oscillates on the rapid time scale $O(\varepsilon)$.

It is interesting to see how this chaotic solution is approached starting from a constant initial function. As shown in Figure 3, there is a transient phase during which the slowly varying plateau/boundary-layer solution is eroded. The mechanism of this erosion is that the structure of the adjoining boundary layers evolves, with and more and more rapid wiggles being added as time progresses.

Some illumination of these observations was provided by Wattis [13]. Integration of (2.1) allows the solution to be written as

$$
x(t)=x(0) \mathrm{e}^{-t / \varepsilon}+\int_{0}^{t / \varepsilon} \mathrm{e}^{-\theta} f[x(t-1-\varepsilon \theta)] \mathrm{d} \theta ;
$$

when written in this form, it illustrates the fact that the differential delay equation is equivalent to a map defined on the continuous functions on $[-1,0]$. Suppose we seek a solution having the boundary-layer characteristics of Figure 1 or Figure 3. Define a sequence of times $t_{n}$ with

$$
t_{n}=t_{n-1}+1+\varepsilon r_{n-1},
$$

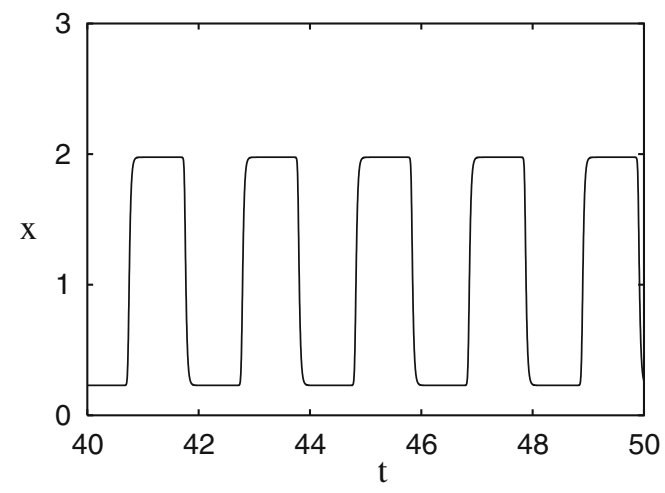

Figure 1. Oscillatory solution of (2.1) using a Hill function (2.6), with $\varepsilon=0.02, \mu=2, n=3$.

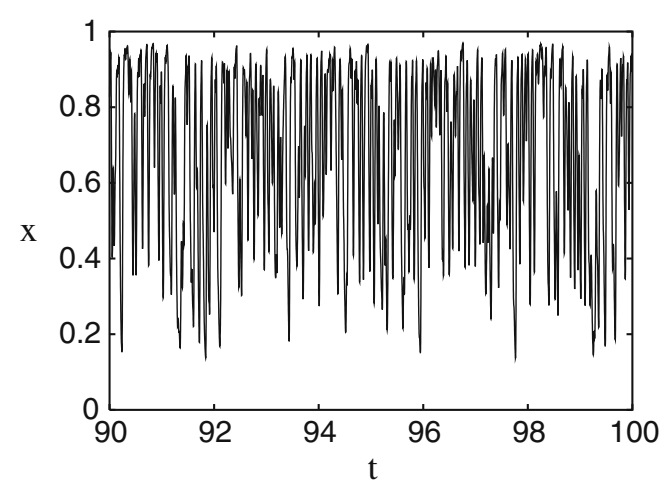

Figure 2. Solution of (2.1) using (2.5) with $\mu=3 \cdot 9, \varepsilon=$ 0.01 . 


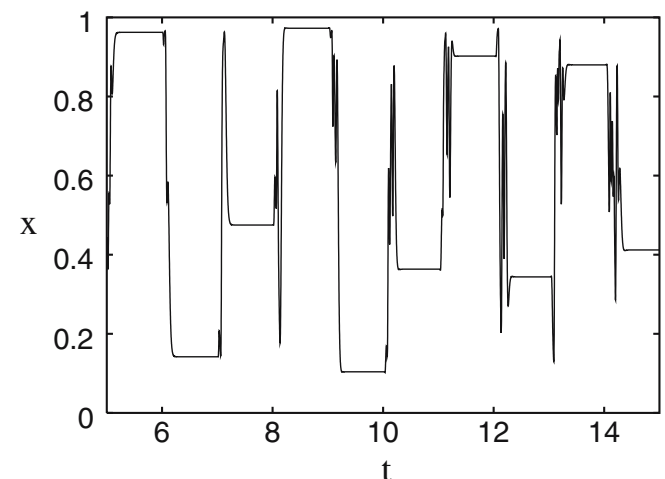

Figure 3. As for Figure 2, but showing part of the initial transient.

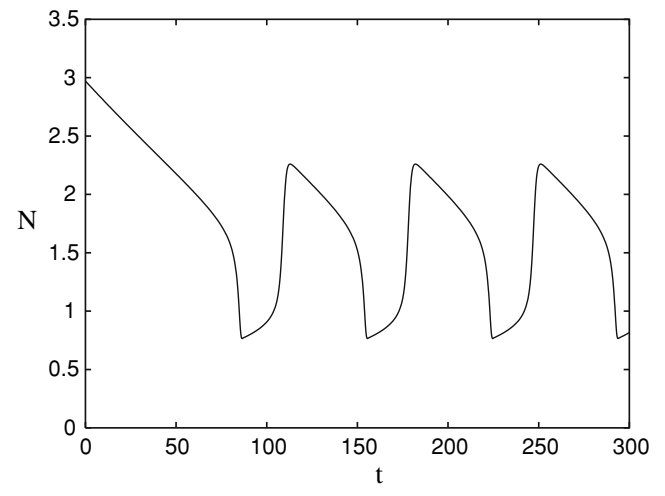

Figure 4. Oscillatory solution of (3.1) when $\varepsilon=0.005$, $\mu=1 \cdot 2, n=3$ and $b=3 \cdot 9$.

and define the functions $\psi_{n}$ via

$$
x\left(t_{n}+\varepsilon \tau\right)=\psi_{n}(\tau) .
$$

The times $t_{n}$ are the switching times where the boundary layers occur, and the functions $\psi_{n}$ are the boundary-layer transition functions.

Substituting these definitions in (2.7) and letting $\varepsilon \rightarrow 0$, we obtain an approximate map, called the 'fast map', relating $\psi_{n}$ to $\psi_{n-1}$ :

$$
\psi_{n}(\tau)=\mathcal{G} \psi_{n-1} \equiv \int_{0}^{\infty} \mathrm{e}^{-\theta} f\left[\psi_{n-1}\left(\tau+r_{n-1}-\theta\right)\right] \mathrm{d} \theta .
$$

The phase shift $r_{n-1}$ can be omitted; it simply recognises the fact that in general there is a phase shift in the solutions of (2.10). For example, the two-cycle shown in Figure 1 corresponds to a two-cycle of the fast map with a suitable choice of $r_{n}=r$.

Although we have presented it slightly differently, the fast map (2.10) is in fact the solution of the local boundary-layer approximation for the transition between plateaux. The essential difference between this and a transition layer in a relaxation van der Pol oscillator is that the boundary-layer equation here is non-local. The boundary layers are spaced in such a way that the solution in one depends on the proceeding one. In an ordinary differential equation, the boundary-layer equation is simpler, and, with luck, solvable. It is also simpler for a delay equation such as that here, but the boundary-layer problem is still fundamentally infinite-dimensional.

This reduction appears to spell the death knell for further asymptotic investigation, because there are no $\varepsilon \mathrm{s}$ left in (2.10). All that we are left with is conjecture. As $\mu$ increases when $\varepsilon=0$, we see a period-doubling sequence in the underlying map, at least for the logistic map. The first of these transitions, from fixed point to two cycle, extends into the $(\mu, \varepsilon)$ plane as a Hopf bifurcation curve $\varepsilon=\varepsilon_{1}(\mu)$. A sinusoidal oscillation exists for $\varepsilon<\varepsilon_{1}$, and as $\varepsilon \rightarrow 0$, this becomes relaxational. Calculation of $\varepsilon_{1}$ indicates that it is an increasing function of $\mu$, suggesting the conjecture that if the underlying map (2.4) has a stable fixed point, then so also does the fast map. It is relatively straightforward to show this, but the extension of the conjecture to higher-order periodic points is less tractable. Nevertheless, we can at least suggest that a similar sequence of bifurcation curves $\varepsilon_{2}(\mu), \varepsilon_{4}(\mu)$, etc., emerge into $\mu>0$ from the period-doubling points on $\mu=0$.

It is less obvious to see how chaotic trajectories of the fast map are created, although numerical evidence indicates that chaotic trajectories of transition functions emerge, as do 
those in the differential delay equation, via the creation of extra loops. As for the asymptotic approximation of a solution such as that in Figure 2, this seems very difficult. While Figure 6 resembles an ordinary relaxation oscillator, that in Figure 2 appears more susceptible to a WKB approximation, but one for a chaotic oscillator, and such methods have not been developed even for ordinary differential equations.

\section{Relaxation oscillations in stem-cell kinetics}

The preceding example resembles a standard singular perturbation problem, but is analytically intractable. A different example, whose behaviour is classically analogous to ordinary differential equation relaxation oscillators, but which appears at first sight to be regular, occurs in a model of stem-cell renewal. The so called $G_{0}$ model of stem-cell renewal is a delay feedback system, and has been described by Mackey $[14,15]$. The model takes the dimensionless form

$$
\left.\dot{N}=g\left(N_{1}\right)-g(N)+\varepsilon\left[\mu g\left(N_{1}\right)-N\right)\right],
$$

in which $N$ represents the stem-cell population, $\dot{N}=\mathrm{d} N / \mathrm{d} t, N_{1} \equiv N(t-1)$ is the delayed term, and

$$
g(N)=b N h(N)
$$

is a controller, where $h(N)$ is usually taken to be a Hill function,

$$
h(N)=\frac{1}{1+N^{n}} .
$$

The model has three dimensionless parameters other than the exponent $n: b$ represents the strength of the controller, $\varepsilon$ represents the rate of loss by differentiation to committed stem cells, and $\mu$ represents the net production of stem cells after cell division, allowing for apoptosis (cell death). Typical inferred values of these parameters are $n=3, b \approx 3.9, \mu \approx 2.6$ and $\varepsilon \approx$ $0 \cdot 11$. For these values, the unique steady state is stable, but if $\mu$ is reduced, then a Hopf bifurcation occurs, and stable periodic behaviour is obtained. Of interest in a variety of blood diseases (see [16]) is that oscillations in blood cell type are often seen, having long periods of the order of 60 days. Since the underlying time scale in the dimensionless $G_{0}$ model is $2 \cdot 2$ days, such long periods indicate a dimensionless period of about 30. A question then arises as to what causes such anomalously long oscillation periods.

The answer to this is the size of the parameter $\varepsilon$. When $\varepsilon=0 \cdot 11, \mu=1 \cdot 2, n=3$ and $b=3 \cdot 9$, the period is about 9.9. As $\varepsilon$ is reduced, we find that the period is of order $1 / \varepsilon$, and the oscillation takes on an increasingly relaxational form, as shown in Figure 4.

It is possible to analyse this oscillation using asymptotic techniques, and this was done by Fowler and Mackey [17]. The treatment parallels that of the relaxation Van der Pol oscillator as expounded in Kevorkian and Cole's book [5, Section 2.6], for example. If we define a slow time

$$
\tau=\varepsilon t,
$$

then we can write the delayed function $N(t-1)=N(\tau-\varepsilon)$ as $N_{\varepsilon}$, and the stem-cell model can be written in the form

$$
N^{\prime}=\frac{g\left(N_{\varepsilon}\right)-g(N)}{\varepsilon}+\mu g\left(N_{\varepsilon}\right)-N,
$$

where $N^{\prime}=\mathrm{d} N / \mathrm{d} \tau$. The assumption that $N$ varies on the time scale $\tau=O(1)$ allows us to expand $N_{\varepsilon}$ in a Taylor expansion about $\tau$, and at leading order (3.5) implies 


$$
N^{\prime}=\frac{\mu g(N)-N}{1+g^{\prime}(N)} .
$$

The behaviour of $N$ in this slow phase depends on the shape of $g$ and the value of $\mu$. From (3.2) and (3.3), we assume that $g$ is unimodal, with a unique positive steady state $N^{*}$ where $N^{*}=\mu g\left(N^{*}\right)$. Oscillations occur when there is a range of $N \in\left(N_{-}, N_{+}\right)$where $g^{\prime}(N)<-1$, and $N^{*} \in\left(N_{-}, N_{+}\right)$. In that case the solution consists of two slow phases in which $N<N_{-}$ and $N>N_{+}$, respectively. The slow approximation (3.6) breaks down as $N \rightarrow N_{-}$or $N \rightarrow N_{+}$, and there is a fast transition layer on the time scale $t \sim O(1)$, in which

$$
\dot{N} \approx g\left(N_{1}\right)-g(N) .
$$

The solution of (3.7) must be obtained numerically, but it does allow the transitional behaviour we seek.

What is interesting about this asymptotic analysis is that it follows that for the van der Pol oscillator very closely. Indeed, a suggestive analogy can be drawn if we define the two functions $v$ and $\hat{v}$ as

$$
v=g\left(N_{\varepsilon}\right)+\varepsilon\left[\mu g\left(N_{\varepsilon}\right)-N\right]
$$

and

$$
\hat{v}=\frac{g(N)-g\left(N_{\varepsilon}\right)}{\varepsilon} .
$$

The Equation (3.1) can then be written in the form

$$
\begin{aligned}
& \varepsilon N^{\prime}=v-g(N), \\
& N^{\prime}+\hat{v}=\mu g(N)-N-\varepsilon \mu \hat{v} .
\end{aligned}
$$

These are not independent, since we may consider $\hat{v}$ to be an infinite-dimensional functional of $v$.

At least on the slowly varying part of the solution where $v \approx g(N)$, we can see that, from (3.9), $\hat{v} \approx v^{\prime}$. If we make the identification $\hat{v}=v^{\prime}$ everywhere, the infinite-dimensional system collapses to a second-order system (3.10), the relaxation oscillations of which are simply studied. The interesting thing is that such an analysis exactly mirrors the analysis of the delay differential equation.

The feature which distinguishes this problem from the preceding one is that the delay acts on the fast time scale: the outer problem is finite-dimensional, and there is no faster time scale than the delay time.

\subsection{TRANSITION Layers AND SIR MODELS}

The main distinction between the analysis of the analogue two-dimensional system (3.10) with $\hat{v}=v^{\prime}$ and the infinite-dimensional system with (3.8) and (3.9) is that the rapid transitions which are becoming visible in Figure 4 are described by the delay-differential equation (back on the $t$ time scale)

$$
\dot{N} \approx g\left(N_{1}\right)-g(N) .
$$

For the asymptotic expansions of the solution, all that is needed of the solution of this equation is the behaviour as $t \rightarrow \pm \infty$. If we assume that $N$ tends to a constant as $t \rightarrow \infty$ (it necessarily does so as $t \rightarrow-\infty$ because of matching requirements), then it is straightforward to show that the values at $\pm \infty$ are related by 


$$
[N+g(N)]_{-\infty}^{+\infty}=0,
$$

but it is less easy to show that $N$ does in fact tend to a constant.

There is an interesting connection between the transition equation (3.11) and the $S I R$ model of epidemiology (see, for example, [18]). The simple form of the SIR model is

$$
\dot{S}=-k S I, \quad \dot{I}=k S I-r I, \quad \dot{R}=r I,
$$

where $S$ represents the susceptible population, $I$ the infected population, and $R$ the recovered, or removed, population.

The first-order decay term $-r I$ is of dubious provenance, as it implies that there is an exponential distribution of infection times, whereas in reality diseases tend to last for a reasonably fixed period. Consideration of this leads us to consider an age-structured model.

Suppose that $i(t, a)$ represents the density (with respect to $a$ ) of infected individuals at time $t$ who have been infected for a time $a$ (i.e., their 'age' of infection is $a$ ). We suppose the rate of removal $r(a)$ is a function of age, so that

$$
\frac{\partial i}{\partial t}+\frac{\partial i}{\partial a}=-r(a) i .
$$

The total infected class is

$$
I=\int_{0}^{\infty} i \mathrm{~d} a,
$$

which on integrating (3.14) implies

$$
\frac{\mathrm{d} I}{\mathrm{~d} t}=-\int_{0}^{\infty} r(a) i \mathrm{~d} a+\left.i\right|_{a=0} .
$$

Evidently $\left.i\right|_{a=0}$ is the rate of addition to the infected class, whence we take

$$
\left.i\right|_{a=0}=k S I \text {, }
$$

and this provides the initial condition for $i$ on $a=0$.

If $r$ is constant we recover (3.13). For $r=r(a)$, we solve (3.14) using the method of characteristics subject to the initial conditions on $a=0$,

$$
t=\eta, \quad i=i_{0}(\eta) \equiv k S(\eta) I(\eta), \quad a=0,
$$

valid for $\eta>0$. (The other part of the initial condition is applied at $t=0, a>0$, but this part of the initial condition only contributes to a transient part of the solution for a finite time.)

The solution of (3.14) is

$$
i=i_{0}(t-a) \exp \left[-\int_{0}^{a} r(s) \mathrm{d} s\right],
$$

valid for $t>a$. For $t<a$, the solution depends on the initial condition at $t=0$, and if, for example, $i=0$ initially, then $i=0$ for $t<a$.

In the particular case where there is a fixed period of infection, $\tau$, we can suppose that $r=0$ for $a<\tau$, but $r$ becomes rapidly infinite as $a$ increases through $\tau$. As a consequence, we have that

$$
\int_{0}^{a} r(s) \mathrm{d} s=\left\{\begin{array}{cc}
0, & a<\tau, \\
\infty, & a>\tau,
\end{array}\right.
$$


and thus

$$
\begin{array}{llrl}
i=i_{0}(t-a), & & a<\tau, \\
i=0, & & a>\tau .
\end{array}
$$

Finally, we have that (for $t>\tau$ )

$$
I=\int_{t-\tau}^{t} i_{0}(s) \mathrm{d} s .
$$

In view of the definition of $i_{0}$, we can deduce from this that

$$
I=-S(t)+S(t-\tau),
$$

and therefore that $S$ satisfies the differential delay equation

$$
\dot{S}=k S\left[S-S_{\tau}\right],
$$

where $S_{\tau}=S(t-\tau)$. This equation can be written in the form (3.11) with time scaled by $\tau$, and $N=-\log S$ and $g(N)=\mathrm{e}^{-N}$.

In Murray's book [19], he shows that successive outbreaks of rabies epizootics can be modelled within the SIR framework by allowing for recovery of the population. Fowler [20] analyses the equivalent model when the disease incubation period has a prescribed distribution. Distributed delay equations are obtained, whose solutions can also be described asymptotically.

\section{Extinction oscillations in the delayed logistic equation}

Gurney et al.'s [8] introduction of the delayed recruitment equation was motivated by the famous Nicholson blowfly experiments [21], in which a confined and resource-limited blowfly population was found to oscillate chaotically. Earlier, May [22] tried to fit the Nicholson data with mixed success using the delayed logistic equation

$$
\dot{y}=\alpha y\left(1-y_{1}\right),
$$

in which $\dot{y}=\mathrm{d} y / \mathrm{d} t$ and $y_{1}=y(t-1)$. This was one of the earliest ecological models to introduce the idea of a delay in the control of a population. Hutchinson [23] modified the logistic equation $\dot{y}=\alpha y(1-y)$ by supposing that the resource-limiting nonlinear term depended on the population at an earlier time, representing the finite gestation time of the population. As a model of resource-limited population growth, the delayed logistic equation was, in its time, a useful paradigm, but it is conceptually suspect, The reason for this is that it states that the population-growth rate depends on current population $y$ but resource (thus $\alpha\left(1-y_{1}\right)$ ) at an earlier time. It is as if the gestation time is zero, but resource digestion time is finite, and hence the delay term. This is unrealistic.

The delayed logistic equation is of mathematical interest because it is one of the simplest examples of a delay equation which has stable periodic solutions. For $\alpha>\pi / 2$, the steady state undergoes a Hopf bifurcation. It is straightforward to apply multiple scale type techniques to analyse the weakly nonlinear oscillation when $\alpha-\pi / 2$ is small, and this was done by Morris [6]. To use multiple scales, one writes $y(t)=y(t, \tau)$, where $\tau=\varepsilon t$, and Taylor expands the delayed terms with respect to the slow time $\tau$. (Morris in fact only used the Poincaré-Lindstedt method.) 
More interesting is the fact that the periodic solutions become clearly relaxational for $\alpha \gtrsim 3$. This relaxational oscillation takes a particular form: the oscillations consist of a series of spikes between which the variable $y$ is close to zero. This kind of behaviour is also exhibited by many other oscillations in systems with and without delay: for example, the Lorenz chaotic oscillations take this form when both parameters $r$ and $\sigma$ are large. A typical numerical solution of (4.1) is shown in Figure 5. It is of interest to try and calculate the asymptotic form of this solution when $\alpha \gg 1$, and to explain why it operates at such apparently low values of $\alpha$.

If asymptotic methods for the delayed recruitment equation appear to be intractable, while those for the stem-cell kinetic are straightforward if lengthy (the difficult part was establishing what the asymptotically small parameter was), the delayed logistic equation appears to require a genuinely novel method of approach. This was done by Fowler [7], and we sketch his method here. In the near zero phases, the solution grows exponentially, thus $y \sim \mathrm{e}^{\alpha t}$. This motivates the introduction of an exponential time

$$
T=\mathrm{e}^{\alpha t},
$$

and a corresponding small parameter

$$
\delta=\mathrm{e}^{-\alpha} .
$$

If we take the time origin for $t$ to be at a pulse, then approximately $y \approx T$ for $T<0$, and this suggests we define $y(t)=T u(T)$, so that

$$
u^{\prime}(T)=-\delta u(T) u(\delta T),
$$

and we suppose $u \rightarrow 1$ as $T \rightarrow 0$. Since the solution is in fact periodic, this asymptotic assumption crucially supposes that the previous pulse occurs when $T$ is exponentially small (in $\delta$ ). This is confirmed a posteriori.

The approximation proceeds by seeking an asymptotic solution $u \sim u_{0}+\delta u_{1}+\cdots$ for $u$, whence (Taylor expanding $u(\delta T)$ about $T=0$, bearing in mind the comment in the preceding paragraph) we find

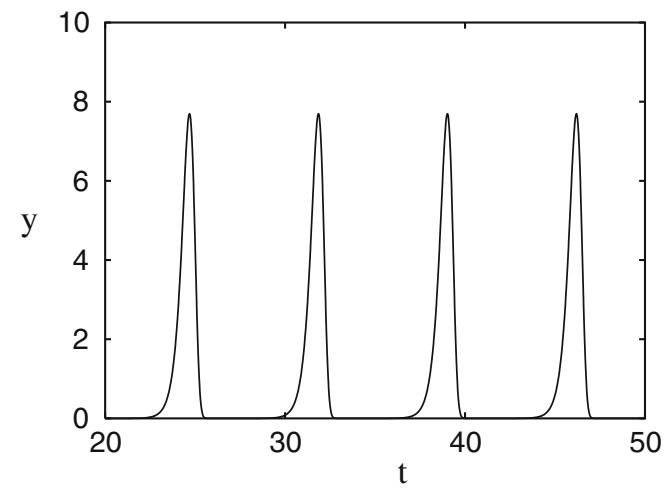

Figure 5. Solution of the delayed logistic equation (4.1) when $\alpha=3$.

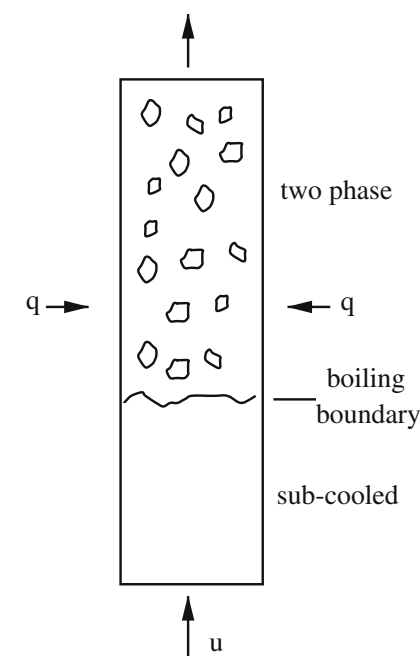

Figure 6. Geometry of heated flow in a boiler tube. 


$$
u \sim 1-\delta T+\cdots
$$

This is invalid when $T \sim 1 / \delta$, thus we define $\tilde{T}=\delta T$, and seek a new expansion. Matching it to the $T \sim 1$ expansion, we find

$$
u \sim \mathrm{e}^{-\tilde{T}}\left[1+\frac{1}{2} \delta \tilde{T}^{2}+\cdots\right],
$$

which we can equivalently write in the form

$$
u \sim \exp \left[-\tilde{T}+\frac{1}{2} \delta \tilde{T}^{2}+\cdots\right]
$$

and in this form we see that the expansion again breaks down when $\tilde{T} \sim 1 / \delta$, and a further expansion is necessary in which $T^{*}=\delta \tilde{T}$, and $u=\exp [-\phi / \delta]$. Expanding the equation and solving for $\phi$, matching to the $\tilde{T} \sim O(1)$ solution, yields

$$
\phi \sim 1-\mathrm{e}^{-T^{*}}+\delta\left[1-\left\{1+T^{*}+\frac{1}{2} T^{* 2}\right\} \mathrm{e}^{-T^{*}}\right]+\cdots .
$$

Luckily no further progression is necessary, because this solution can be used to match to the next pulse. If the period of $y$ (in $t$ ) is $P$, then this requires

$$
\mathrm{e}^{-\alpha P} u\left(T \mathrm{e}^{-\alpha P}\right)=u(T),
$$

and using this to match the $T^{*}$ solution to the next pulse leads to

$$
P=\frac{1}{\alpha}\left[\mathrm{e}^{\alpha}+1+O\left(\mathrm{e}^{-\alpha}\right)\right],
$$

which is found to agree excellently with numerical results for $\alpha$ greater than about 2.5. (Note that the Hopf bifurcation only occurs at $\alpha \approx 1.57$ !)

Here we have a problem with a large delay, where the solution involves a completely different kind of method. The delay is much shorter than the period, but the three segments of the solution all operate on the delay time scale. Crucially, the delay is again the shortest time scale of relevance. Incidentally, there is nothing in this method which requires periodicity of the solution, since the method in effect determines a Poincare map. Chaotic solutions of similar equations with the same occurrence of sporadic outbreaks would be susceptible to the same analysis.

\section{Density-wave oscillations in boilers}

Density-wave oscillations in two-phase flow have been of concern in the nuclear industry for a long time. In a steam-generating boiler, water in an array of pipes is heated externally, and begins to boil as it flows along the pipes. In certain situations, the resulting two-phase flow can be oscillatorily unstable, which is an undesirable feature in industrial systems. The instability mechanism is the same one that produces chugging in a domestic back boiler, if the pipework to the hot-water tank is incorrectly installed (i.e., a section of pipe from fire to hotwater tank is inclined downwards), and also the same that produces geysering in geothermal springs. A more direct analogy is in certain types of effusive volcanic eruptions, such as that at Villarrica volcano in Chile, where the magma flow in the vent appears to oscillate between a bubbly-flow régime and a slug-flow régime [24].

The simplest model to describe the instability was posed and analysed by Davies and Potter [25]. They studied a homogeneous two-phase flow model, in which it is assumed that in the two-phase region, the liquid and vapour phases move with the same velocity. This is 
the simplest assumption, though it is inaccurate, particularly in the annular flow régime. Nevertheless it appears to capture the essence of the instability, and serves as a useful starting point. There have been a good number of other theoretical investigations of the problem. Many of these have been reviewed by Aldridge and Fowler [26]. A recent contribution is that by Delmastro et al. [27].

The geometry of the flow under consideration is shown in Figure 6. We denote the vertical coordinate along the tube as $x$, so that the tube occupies $0<x<l$. The flow is supposed turbulent, as is generally the case, and we use a cross-sectionally averaged model of the turbulent flow, with the effects of Reynolds stresses modelled by an empirical wall-friction factor. Heat is added to the flow at a rate $q$, and we suppose that the flow consists of two régimes, a sub-cooled region occupying the initial part of the tube $0<x<r(t)$, and a two-phase region $r<x<l$, in which liquid and vapour coexist as a bubbly flow. In practice, such two-phase flows pass through a succession of flow régimes, from bubbly to slug to churn to annular, as the vapour fraction increases. To model such different régimes at all requires a more sophisticated two fluid model, but it is not known with any theoretical confidence what controls the transition between the different régimes, so that the effort involved may not be appropriate.

We follow Davies and Potter [25] and Fowler [28] in posing a homogeneous model of the two-phase region. We suppose that the flow is driven by a pressure drop $\Delta p$, and that the inlet temperature and pressure (and thus also enthalpy) is prescribed. Equations describing the flow are those of conservation of mass, momentum and energy, and they take the form

$$
\begin{aligned}
& \rho_{t}+(\rho u)_{x}=0, \\
& \rho\left(u_{t}+u u_{x}\right)=-p_{x}-\rho g-\frac{4 f \rho u^{2}}{d}, \\
& \rho \frac{\mathrm{d} h}{\mathrm{~d} t}-\frac{\mathrm{d} p}{\mathrm{~d} t}=\frac{4 q}{d},
\end{aligned}
$$

where $\rho$ is density (of the liquid in the single-phase region, of the mixture in the twophase region), $u$ is velocity, $p$ is pressure, and $h$ is enthalpy; $\frac{\mathrm{d}}{\mathrm{d} t}$ is the material derivative $\frac{\partial}{\partial t}+u \frac{\partial}{\partial x}, f \rho u^{2}$ is the wall stress, $q$ is the heat input per unit surface area, $g$ is the gravitational acceleration, and $d$ is the tube diameter. These are to be solved subject to the conditions

$$
\begin{aligned}
& h=h_{0} \quad \text { at } \quad x=0, \\
& -\int_{0}^{l} p_{x} \mathrm{~d} x=\Delta p .
\end{aligned}
$$

One simplification which we immediately make is to suppose that

$$
\frac{\Delta p}{\rho_{g} L} \ll 1
$$

where $\rho_{g}$ is vapour density and $L$ is latent heat. This is well satisfied in industrial contexts, and guarantees that the adiabatic pressure derivative term in the enthalpy equation can be ignored, which we henceforth do. For steam-water systems, this is a good approximation. For example, $\rho_{g} L \approx 270$ bars $\left(1 \mathrm{bar}=10^{5} \mathrm{~Pa}=10^{5} \mathrm{~N} \mathrm{~m}^{-2}\right)$ at an operating pressure of 30 bars, and $\rho_{g} L \approx 13$ bars at atmospheric pressure (where we take vapour density at the boiling point). 


\subsection{SUB-COOLED REGION}

In the single phase sub-cooled liquid near the inlet, we suppose the liquid is incompressible, so that $\rho=\rho_{l}$ is constant, and thus $u=U(t)$, and

$$
h_{t}+U h_{x}=\frac{4 q}{\rho_{l} d} .
$$

With the inlet condition $(5.2)_{1}$, this is easily solved, to find

$$
\begin{aligned}
& x=\int_{\tau}^{t} U(\theta) \mathrm{d} \theta, \\
& h=\frac{4 q}{\rho_{l} d}(t-\tau)+h_{0} .
\end{aligned}
$$

We assume here that $q$ is constant. A more realistic assumption is to have $q$ depend on $u$, and it is possible to treat this case also, though less easily. We define the saturation enthalpy of the liquid at the boiling point to be $h_{l}$, and we define the inlet sub-cooling to be

$$
\Delta h_{0}=h_{l}-h_{0} ;
$$

then $h=h_{l}$ defines the location of the boiling boundary $x=R(t)$, and we find

$$
R(t)=\int_{t-\tau_{0}}^{t} U(\theta) \mathrm{d} \theta,
$$

where

$$
\tau_{0}=\frac{\rho_{l} d \Delta h_{0}}{4 q} .
$$

Note that (5.7) introduces a delay $\tau_{0}$ into the system: the boiling boundary position $R(t)$ depends on the history of the inlet velocity $U(t)$.

\subsection{TWO-PHASE REGION}

In the two phase liquid-vapour region $x>R$, we define the void fraction $\alpha$ to be the volume fraction of vapour. We then have the definitions of two phase density and enthalpy:

$$
\begin{aligned}
& \rho=\rho_{l}(1-\alpha)+\rho_{g} \alpha, \\
& \rho h=\rho_{l} h_{l}(1-\alpha)+\rho_{g} h_{g} \alpha,
\end{aligned}
$$

where suffixes $l$ and $g$ indicate liquid and gas properties; note that the latent heat is

$$
L=h_{g}-h_{l} .
$$

Eliminating $\alpha$ yields $h$ as a function of $\rho$, and we substitute this in (5.1) to find that $\rho$ and $u$ in $x>R$ satisfy the equations

$$
\begin{aligned}
& \rho_{t}+u \rho_{x}=-u_{x} \rho, \\
& \frac{\rho_{g} \rho_{l} L}{\Delta \rho} u_{x}=\frac{4 q}{d},
\end{aligned}
$$

where

$$
\Delta \rho=\rho_{l}-\rho_{g},
$$

subject to

$$
\rho=\rho_{l}, \quad u=U(t) \quad \text { on } \quad x=R .
$$




\section{A.C. Fowler}

When this pair of equations is solved, then the inlet velocity is found by requiring that

$$
\begin{aligned}
\Delta p= & \frac{4 f}{d}\left[\rho_{l} U^{2} R+\int_{R}^{l} \rho u^{2} \mathrm{~d} x\right]+g\left[\int_{R}^{l} \rho \mathrm{d} x+\rho_{l} R\right] \\
& +\left.\rho u^{2}\right|_{l}-\rho_{l} U^{2}+\frac{\partial}{\partial t} \int_{R}^{l} \rho u \mathrm{~d} x+\rho_{l} \dot{U} R .
\end{aligned}
$$

\subsection{NON-DIMENSIONALISATION}

At this point it is convenient to non-dimensionalise the model. We diverge from Fowler [28], who scaled his equivalent model in a less mature fashion. We scale the variables as follows:

$$
\rho \sim \rho_{l}, \quad x, R \sim l, \quad t \sim \tau_{0}, \quad u, U \sim u_{0}=\frac{l}{\tau_{0}} ;
$$

we then find the dimensionless model to be

$$
\begin{aligned}
R= & \int_{t-1}^{t} U(\theta) \mathrm{d} \theta \\
\Delta p= & \Delta p_{f}\left[U^{2} R+\int_{R}^{1} \rho u^{2} \mathrm{~d} x\right]+\Delta p_{g}\left[\int_{R}^{1} \rho \mathrm{d} x+R\right] \\
& +\Delta p_{i}\left[\left.\rho u^{2}\right|_{1}-U^{2}+\frac{\partial}{\partial t} \int_{R}^{1} \rho u \mathrm{~d} x+\dot{U} R\right]
\end{aligned}
$$

where

$$
\Delta p_{i}=\rho_{l} u_{0}^{2}, \quad \Delta p_{f}=\frac{4 f l \rho_{l} u_{0}^{2}}{d}, \quad \Delta p_{g}=\rho_{l} g l,
$$

and $\rho$ and $u$ satisfy

$$
\begin{aligned}
& \rho_{t}+u \rho_{x}=-u_{x} \rho, \\
& \varepsilon u_{x}=1
\end{aligned}
$$

in $R<x<1$, with

$$
u=U, \quad \rho=1 \quad \text { on } \quad x=R
$$

and

$$
\varepsilon=\frac{\rho_{g} L}{\Delta \rho \Delta h_{0}} .
$$

For reasons which will emerge, we define the pressure drop scale

$$
\Delta p_{0}=\frac{\Delta p_{f}}{\varepsilon}=\frac{4 f l \rho_{l} u_{0}^{2} \Delta \rho \Delta h_{0}}{\rho_{g} L d}
$$

and then in terms of the dimensionless parameters

$$
\gamma=\frac{\Delta p_{g}}{\Delta p_{f}}=\frac{g d}{4 f u_{0}^{2}}, \quad \delta=\frac{\Delta p_{i}}{\Delta p_{f}}=\frac{d}{4 f l},
$$

the dimensionless pressure drop $\Pi=\Delta p / \Delta p_{0}$ is 


$$
\begin{aligned}
\frac{\Pi}{\varepsilon}=\left[U^{2} R\right. & \left.+\int_{R}^{1} \rho u^{2} \mathrm{~d} x\right]+\gamma\left[\int_{R}^{1} \rho \mathrm{d} x+R\right] \\
+\delta & {\left[\left.\rho u^{2}\right|_{1}-U^{2}+\frac{\partial}{\partial t} \int_{R}^{1} \rho u \mathrm{~d} x+\dot{U} R\right] . }
\end{aligned}
$$

The solution for $u$ is evidently

$$
u=U+\frac{x-R}{\varepsilon} .
$$

The characteristic solution for $\rho$ is then found to be, after some rearrangement and integration by parts,

$$
\begin{aligned}
& x=R(t)+\varepsilon \int_{0}^{s} U_{1}(t-\varepsilon \zeta) \mathrm{e}^{\zeta} \mathrm{d} \zeta, \\
& \rho=\mathrm{e}^{-s},
\end{aligned}
$$

where

$$
U_{1}(\theta)=U(\theta-1),
$$

and note that then

$$
u=U+\int_{0}^{s} U_{1}(t-\varepsilon \zeta) \mathrm{e}^{\zeta} \mathrm{d} \zeta
$$

\subsection{A REDUCED MODEL}

The model thus reduces to the pair of Equations (5.23) and (5.16) , with the various integrals computed using (5.25) and (5.27). Writing them together, we obtain

$$
\begin{aligned}
& R=\int_{t-1}^{t} U(\theta) \mathrm{d} \theta \\
& \frac{\Pi}{\varepsilon}=\left[U^{2} R+\int_{R}^{1} \rho u^{2} \mathrm{~d} x\right]+\gamma\left[\int_{R}^{1} \rho \mathrm{d} x+R\right]+\delta\left[\left.\rho u^{2}\right|_{1}-U^{2}+\frac{\partial}{\partial t} \int_{R}^{1} \rho u \mathrm{~d} x+\dot{U} R\right]
\end{aligned}
$$

in which $\rho$ and $u$ are defined by

$$
\begin{aligned}
& \rho=\mathrm{e}^{-s}, \\
& u=U+\int_{0}^{s} U_{1}(t-\varepsilon \zeta) \mathrm{e}^{\zeta} \mathrm{d} \zeta
\end{aligned}
$$

and $s$ is defined through

$$
x=R(t)+\varepsilon \int_{0}^{s} U_{1}(t-\varepsilon \zeta) \mathrm{e}^{\zeta} \mathrm{d} \zeta .
$$

The delay (scaled to be one) in this system is explicitly represented by the delayed function $U_{1}(t)=U(t-1)$, but a second delay is manifested through the dependence of $u$ and $x$ on the delayed values of $U_{1}$.

The model depends on the four parameters $\Pi, \varepsilon, \gamma$ and $\delta$. In the literature (see, for example $[29,30])$ it is common to describe the behaviour of the system in terms of two dimensionless parameters known as the sub-cooling number $N_{\text {sub }}$ and the phase change number $N_{\text {pch. }}$. In terms of the parameters defined above, these are defined as (see, for example, [26]) by

$$
N_{\text {sub }}=\frac{1}{\varepsilon}, \quad N_{\text {pch }}=\frac{1}{\varepsilon U} .
$$


Stability diagrams are often given in terms of these parameters. (Obviously, assuming that $U$ is constant, i.e., under steady-state conditions.)

It is generally the case that $\rho_{g} \ll \rho_{l}$, and we will suppose that $\Delta h_{0} \sim L$, so that the inlet water is decently sub-cooled. It then follows that $\varepsilon \ll 1$, a typical value at an operating pressure of 30 bars being $\varepsilon \approx 0.02$. As illustrative values, we take $u_{0}=1 \mathrm{~m} \mathrm{~s}^{-1}, \rho_{l}=10^{3} \mathrm{~kg} \mathrm{~m}^{-3}$, $l=10 \mathrm{~m}, d=10^{-2} \mathrm{~m}, f=0.01, g \sim 10 \mathrm{~m} \mathrm{~s}^{-2}$. From these we find $\gamma \approx 2.5, \delta \approx 0.025$. Thus it seems reasonable to suppose that $\gamma \sim O(1)$, and $\delta \sim \varepsilon \ll 1$.

We see from (5.25) that if $0<R<1$ and is not close either to the inlet or outlet, then $s_{1} \sim$ $\log (1 / \varepsilon)$, where $s=s_{1}$ when $x=1$. Since we then have that $\varepsilon \zeta \sim \varepsilon \log (1 / \varepsilon) \ll 1$, we can expand the integral terms in (5.23), and at leading order we then find that $s_{1}$ is given by

$$
s_{1} \sim \log \left[\frac{1-R}{\varepsilon U_{1}}\right],
$$

and the dimensionless pressure drop $\Pi$ can be written as

$$
\begin{aligned}
\Pi \approx & \frac{1}{2} U_{1}(1-R)^{2}+\varepsilon U^{2} R+\varepsilon \gamma\left[\varepsilon U_{1} \log \left(\frac{1-R}{\varepsilon U_{1}}\right)+R\right] \\
& +\delta\left[U_{1}(1-R)-\varepsilon U^{2}\right]+\varepsilon \delta\left[\frac{\mathrm{d}}{\mathrm{d} t}\left\{U_{1}(1-R)\right\}+\dot{U} R\right] .
\end{aligned}
$$

\subsection{Steady states}

Figure 7 shows steady states of $U$ as a function of applied pressure drop. Evidently multiple steady states occur. The middle branch is unstable, this instability being known as Ledinegg instability [31]. In the steady state $R=U$, so that the Ledinegg values $0.44 \lesssim U \lesssim 0.86$ (in Figure 7) correspond to situations where the boiling boundary approaches the outlet.

\subsection{INSTABILITY AND ILL-POSEDNESS}

Oscillatory instabilities can occur as well as the direct Ledinegg instability. To study these we begin by putting $\varepsilon=0$ (now we can see why the factor $\varepsilon$ was included in the denominator of the left-hand side of (5.33)). At leading order, the model reduces to

$$
\Pi \approx \frac{1}{2} U_{1}(1-R)^{2}, \quad R=\int_{t-1}^{t} U(\theta) \mathrm{d} \theta .
$$

This innocuous-looking delay integral equation is ill-posed. (It also appears not to be accurate, at least when $U$ approaches one, since in the steady state $\Pi$ then approaches zero, unlike the full curve shown in Figure 7. We can see the ill-posedness as follows. If we define

$$
W=\int^{t} U(\theta) \mathrm{d} \theta
$$

then $R=W-W_{1}$, and (5.34) is

$$
\dot{W}_{1}=\frac{2 \Pi}{\left(1-W+W_{1}\right)^{2}},
$$

which is an equation of advanced type, i.e., a delay equation with negative delay. In assessing the instability of the steady state, we then find an infinite number of unstable states, whose (complex) growth rate $\sigma$ tends to infinity in the complex plane with $\mathfrak{R e} \sigma>0$.

To be specific, denote the steady state as $R=U=U^{*}$, and linearise the reduced model (5.34). The solutions are exponential, thus 


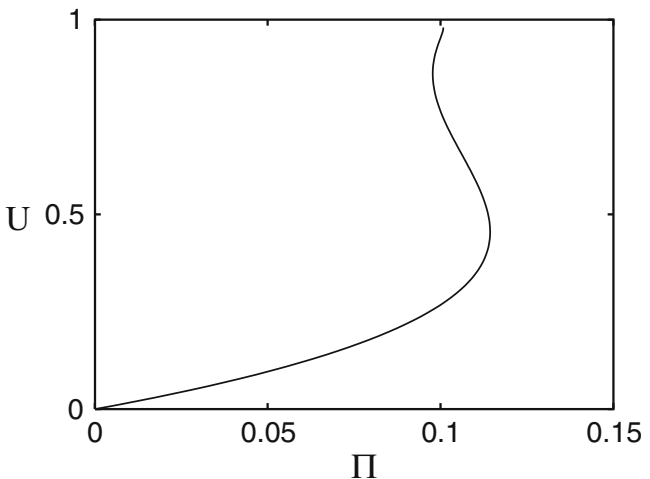

Figure 7. Multiple steady states of (5.33) when $\varepsilon=$ $0.03, \gamma=2.5, \delta=0.025$.

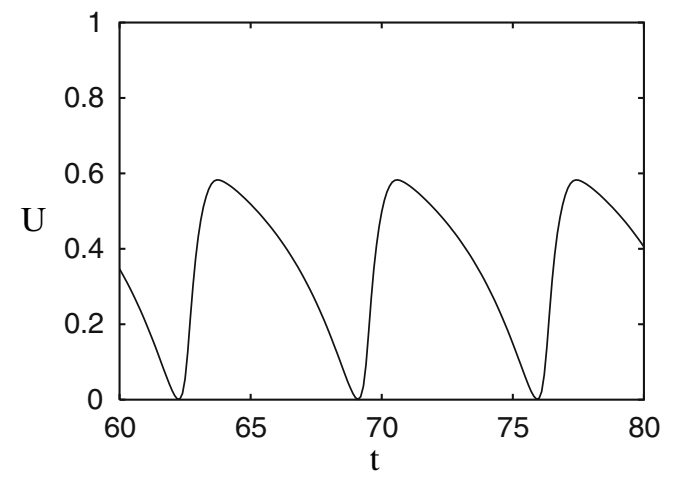

Figure 8. Solution of (5.48) with $\gamma=2.5, \delta=0.025, \varepsilon=$ $0.03, \Pi=0.08$, and $\nu=0.88$.

$$
U-U^{*}=\mathrm{e}^{\sigma t}, \quad R-U^{*}=\frac{1}{\sigma}\left(1-\mathrm{e}^{-\sigma}\right) \mathrm{e}^{\sigma t},
$$

and we require $\sigma$ to satisfy the transcendental equation

$$
g_{0}(\sigma)=\frac{1}{2}\left(1-U^{*}\right)^{2} \mathrm{e}^{-\sigma}-\frac{U^{*}\left(1-U^{*}\right)}{\sigma}\left(1-\mathrm{e}^{-\sigma}\right)=0 .
$$

It is straightforward to show that this equation has an infinite number of roots in the complex plane, and these tend to the essential singularity at $\infty$ in the right half plane, $\mathfrak{R e} \sigma>0$. This accumulation of rapidly growing modes is the signal of an ill-posed equation of advance.

Putting $\varepsilon=0$ is thus a singular approximation. There are a number of small terms in (5.33) which might regularise the model. The principal suspect in this regard is the sub-cooled frictional pressure drop. Adding this yields the model

$$
\Pi \approx \frac{1}{2} U_{1}(1-R)^{2}+\varepsilon U^{2} R .
$$

This gives an equation of mixed type, and regularises the model if $\varepsilon$ is large enough. Linear stability of the steady state yields the equation for the growth rate $\sigma$ as

$$
g_{\varepsilon}(\sigma)=g_{0}(\sigma)+\varepsilon U^{* 2}\left[2+\frac{1-\mathrm{e}^{-\sigma}}{\sigma}\right]=0 .
$$

Of concern is the sector where $\sigma \rightarrow \infty$. It is easy to show that $\mathfrak{R e} \sigma$ cannot tend to $+\infty$. If $\mathfrak{R e} \sigma$ is bounded, then we find

$$
\sigma \approx \log \left[\frac{\left(1-U^{*}\right)^{2}}{4 \varepsilon U^{*}}\right] \pm(2 n+1) \mathrm{i} \pi
$$

for large integer $n$, and thus the model is regularised if

$$
\varepsilon>\frac{\left(1-U^{*}\right)^{2}}{4 U^{*}} .
$$

This conditional regularisation of the model is reminiscent of the conditional regularisation of two fluid models of bubbly flow for low enough void fraction, associated (perhaps) with flow régime transition boundaries [32]. 
The fact that the regularisation is conditional, and in particular does not apply for sufficiently small $\varepsilon$, suggests that a further regularisation is necessary. The correct term to include is the derivative term, thus we replace (5.39) by

$$
\Pi \approx \frac{1}{2} U_{1}(1-R)^{2}+\varepsilon U^{2} R+\nu R \dot{U},
$$

where

$$
\nu=\varepsilon \delta .
$$

This unequivocally regularises the model. Linear stability of the steady state is determined by values of $\sigma$ for which

$$
g_{\varepsilon, \nu}(\sigma)=g_{\varepsilon}(\sigma)+\nu U^{*} \sigma=0 .
$$

Now as $\sigma \rightarrow \infty$, we must have

$$
\nu U^{*} \sigma+\frac{1}{2}\left(1-U^{*}\right)^{2} \mathrm{e}^{-\sigma} \approx 0,
$$

and it follows from this that

$$
\sigma \approx 2 n i \pi-\log 2 n \pi+\cdots
$$

for large integer $n$. The problem is thus regularised, although of course there will be many unstable modes with smaller $|\sigma|$.

To write an approximate model, we retain the regularising term $v R \dot{U}$, but ignore the other term in $v$, which allows us to write the model as a pair of delay differential equations:

$$
\begin{aligned}
& \nu R \dot{U}=\Pi-\frac{1}{2} U_{1}(1-R)^{2}-\varepsilon U^{2} R-\varepsilon \gamma\left[\varepsilon U_{1} \log \left(\frac{1-R}{\varepsilon U_{1}}\right)+R\right]-\delta\left[U_{1}(1-R)-\varepsilon U^{2}\right], \\
& \dot{R}=U-U_{1} .
\end{aligned}
$$

Although it appears that a useful further approximation can be made by putting the terms in $\varepsilon$ and $\delta$ to zero, this is not numerically accurate, because in practice (see Figure 7) $\Pi$ is small.

Equation (5.48) has the appearance of a singularly perturbed delay differential equation, of the type we have been considering. Given the range of behaviour we have now seen from similarlooking equations, it appears to be a challenge to find asymptotic solutions of (5.48). However, this system has an added twist. If we fix the parameters $\varepsilon=0.03, \delta=0.025, \gamma=2.5, \Pi=0.08$, and progressively reduce $v$, we find that the steady state at $\Pi=0.08, U=0.181$ (see Figure 7) has a Hopf bifurcation to a stable limit cycle as $v$ is reduced through 0.984. As $v$ decreases, the amplitude grows until at $v=0.88$ the inlet velocity decreases to zero (see Figure 8), and for $v<0.88$, reversed flow occurs. The model is not applicable for reversed flow, and indeed the numerical solution breaks down in that case. This appears consistent with theoretical stability results of many authors (see [26]), which indicate that instability is commonplace at small $\varepsilon$. The twist here is that the model itself precludes attainment of the relevant asymptotic limit.

\section{Conclusions}

Classical expositions of singular perturbation methods for differential equations are typically organised by reference to different kinds of examples: boundary layers and matched asymptotic expansions are applied to singularly perturbed boundary-value problems, WKB methods are applied to rapidly oscillating solutions, multiple-scale methods are used for weakly nonlinear oscillators, and so on. 
Delay equations pose particularly severe problems for the application of singular perturbation methods, for two reasons. The first is that such equations are, in general, hard to deal with. The second is that it appears to be very difficult to classify problems into one or other type. Of course, both these problems are aggravated by the paucity of problems in which a successful analysis has been performed.

In this paper, we have brought together a limited but varied set of relatively simply stated differential delay equations, each containing a small parameter which perturbs the solutions in a singular way. We have endeavoured to provide methods of solution, and we have tried in a limited way to identify types of behaviour.

In some situations, such as the stem-cell model (3.1), the application of singular perturbation methods is relatively straightforward, and there is a very clear analogy with the treatment of relaxation oscillations in ordinary differential equations. However, the class of model for which this technique works does not appear to resemble the classical form of a relaxation oscillator, unless one draws an analogy using (3.10).

In contrast, delay equations which do superficially resemble a normal singularly perturbed problem can lead to radically unmanageable 'reduced' problems (the delay recruitment equation) which may or may not be tractable, or to slightly wild asymptotic methods (the delayed logistic equation), which are tractable, but in ways which are wholly unlike classical procedures.

The final and substantive part of this paper concerns the study of a particular problem of interest in industrial applications, namely the occurrence of 'density wave' oscillations in heated two-phase flows. Such problems have been of theoretical and practical interest for more than half a century, but few recognisable theoretical advances have been made. In part, this stems from the notorious difficulty of modelling two-phase flows satisfactorily, and in part because the resulting models come in the form of well-disguised delay equations. For the simplest, homogeneous flow model, we have used direct asymptotic methods to reduce the problem to a pair of delay integral equations (5.34), which are equivalent to a single firstorder differential delay equation (5.36) of advanced type, that is to say, the delay is negative. It is well known that such equations are ill-posed.

In an effort to rescue the model from this fate, we introduced a singularly perturbed term, but then found that the model formulation breaks down well outside the realistic range of parameter values, because of reversed flow at the inlet to the tube. Whether this is a realistic breakdown is unclear, although it is reminiscent of the phenomenon of geysering, in which similar extreme fluctuations occur.

It seems clear that a categorisation of asymptotic methods for delay equations will not be as simple as for ordinary differential equations. Asymptotic methods will have to be used on a case by case basis in the immediate future. Because of this, their application in practical problems will continue to be a difficult, interesting and exciting challenge for the applied mathematician.

\section{Acknowledgement}

I acknowledge the continuing support of my research by the University of Limerick.

\section{References}

1. R.D. Driver, Ordinary and Delay Differential Equations. New York: Springer-Verlag (1977) ix $+501 \mathrm{pp}$

2. J.K. Hale and S.M. Verduyn Lunel, Introduction to Functional-Differential Equations; Volume 99 of Applied Mathematical Sciences. New York: Springer-Verlag (1993) x+447 pp. 
3. O. Diekmann, S.A. van Gils, S.M. Verduyn Lunel and H.-O. Walther, Delay Equations: Functional, Complex, and Nonlinear Analysis; Volume 110 of Applied Mathematical Sciences. New York: Springer-Verlag (1995) xii+534 pp.

4. C.M. Bender and S.A. Orszag, Advanced Mathematical Methods for Scientists and Engineers. New York: McGraw-Hill Book Co. (1978) xiv+593 pp.

5. J. Kevorkian and J.D. Cole, Perturbation Methods in Applied Mathematics; Volume 34 of Applied Mathematical Sciences. New York: Springer-Verlag (1981) x+558 pp.

6. H.C. Morris, A perturbative approach to periodic solutions of delay-differential equations. J. Inst. Math. Appl. 18 (1976) 15-24.

7. A.C. Fowler, An asymptotic analysis of the delayed logistic equation when the delay is large. IMA J. Appl. Math. 28 (1982) 41-49.

8. W.S.C. Gurney, S.P. Blythe and R.M. Nisbet, Nicholson's blowflies revisited. Nature 287 (1980) 17-21.

9. M.C. Mackey and L. Glass, Oscillations and chaos in physiological control systems. Science 197 (1977) 287-289.

10. J. Keener and J. Sneyd, Mathematical Physiology; Volume 8 of Interdisciplinary Applied Mathematics. New York: Springer-Verlag (1998) xx+766 pp.

11. S.-N. Chow and J. Mallet-Paret, Singularly perturbed delay-differential equations. In: Coupled Nonlinear Oscillators (Los Alamos, N.M., 1981); Volume 80 of North-Holland Math. Stud. Amsterdam North-Holland (1983) pp. 7-12.

12. J. Mallet-Paret and R.D. Nussbaum, Boundary layer phenomena for differential-delay equations with statedependent time lags. III. J. Diff. Equ. 189 (2003) 640-692.

13. J.A.D. Wattis, Bifurcations and chaos in a differential-delay equation. Dissertation, M. Sc. in mathematical modelling and numerical analysis Oxford University (1990).

14. M.C. Mackey, A unified hypothesis for the origin of aplastic anemia and periodic haematopoiesis. Blood 51 (1978) 941-956.

15. M.C. Mackey, Mathematical models of hematopoietic cell replication and control. In: M.A.L.H.G. Othmer, F.R. Adler and J.C. Dallon (eds), The Art of Mathematical Modelling: Case Studies in Ecology, Physiology and Biofluids. New Jersey Prentice-Hall (1997) pp. 149-178.

16. C. Haurie, D.C. Dale and M.C. Mackey, Cyclical neutropenia and other periodic hematological disorders: a review of mechanisms and mathematical models. Blood 92 (1998) 2629-2640.

17. A.C. Fowler and M.C. Mackey, Relaxation oscillations in a class of delay differential equations. SIAM J. Appl. Math. 63 (2002) 299-323.

18. J.D. Murray, Mathematical Biology. I: An Introduction; Volume 17 of Interdisciplinary Applied Mathematics. New York: Springer-Verlag (2002) xxiv+551 pp.

19. J.D. Murray, Mathematical Biology. II: Spatial Models and Biomedical Applications; Volume 18 of Interdisciplinary Applied Mathematics. New York: Springer-Verlag (2003) xxvi+811 pp.

20. A.C. Fowler, The effect of incubation time distribution on the extinction characteristics of a rabies epizootic. Bull. Math. Biol. 62 (2000) 633-655.

21. A.J. Nicholson, An outline of the dynamics of animal populations. Aust. J. Zool. 2 (1954) 9-65.

22. R.M. May, Stability and Complexity in Model Ecosystems. Princeton, N. J.: Princeton University Press (1973) $292 \mathrm{pp}$.

23. G.E. Hutchinson, Circular causal systems in ecology. Ann. N. Y. Acad. Sci. 50 (1948) 221-240.

24. E. Calder, private communication.

25. A.L. Davies and R. Potter, Hydraulic stability: an analysis of the causes of unstable flow in parallel channels. Symposium on Two-Phase Flow Dynamics. Eindhoven EUR4288e (1967) pp. 1225-1266.

26. C.J. Aldridge and A.C. Fowler, Stability and instability in evaporating two-phase flows. Surveys Math. Indust. 6 (1996) 75-107.

27. D. Delmastro, L. Juanicó and A. Clausse, A delay theory for boiling flow stability analysis. Int. J. Multiphase Flow 27 (2001) 657-671.

28. A.C. Fowler, Linear and nonlinear stability of heat exchangers. J. Inst. Math. Appl. 22 (1978) 361-382.

29. J.L. Achard, D.A. Drew and R.T. Lahey, The effect of gravity and friction on the stability of boiling flow in a channel. AIChE Symp. Ser. 199 (1980) 104-115.

30. R.C. Dykhuizen, R.P. Roy and S.P. Kalra, Two-fluid model simulation of density-wave oscillations in a boiling flow system. Nucl. Sci. Engng. 94 (1986) 167-179.

31. M. Ledinegg, Instability of flow during natural and forced circulation. Die Wärme 61 (1938) 891-898.

32. C. Pauchon and S. Banerjee, Interphase momentum interaction effects in the averaged multifield model. Part I: void propagation in bubbly flows. Int. J. Multiphase Flow 12 (1986) 559-573. 